\title{
¿QUÉ SIGNIFICA DESEMPEÑO (SUPERIOR) EN ADMINISTRACIÓN ESTRATÉGICA?
}

\author{
WHAT DOES (SUPERIOR) PERFORMANCE MEAN IN STRATEGIC MANAGEMENT? \\ Oswaldo Chacón-Fiallo $\bullet$ Laura Zapata-Cantú ${ }^{b}$ \\ Clasificación: Trabajo conceptual \\ Recibido: 27 -junio- 2020 / Revisado: 27-octubre- 2020; 30 -noviembre- 2020 / Aceptado: 3 -diciembre- 2020
}

\section{Resumen}

El propósito de este artículo es dilucidar la noción de desempeño (firm performance) en las escuelas más representativas de la administración estratégica (Strategic Management) y contribuir mediante una definición y un modelo conceptual integrador para su comprensión de forma multidimensional y multinivel. A través de una revisión de la literatura, en la que se incluyen los trabajos seminales -teóricos y empíricos- de las perspectivas de organización industrial, recursos y capacidades, capacidades dinámicas y basadas en el conocimiento se identifica el tratamiento del constructo desempeño. Posteriormente, se establecen similitudes y diferencias, cuyo resultado se presenta en un modelo conceptual integrador. En administración estratégica, se ha utilizado la expresión firm performance para explicar las consecuencias de la ejecución de la estrategia. Sin embargo, en la revisión de la literatura se ha encontrado que el desempeño como constructo presenta ambigüedad en su definición y falta de delimitación epistemológica, lo cual dificulta su comprensión, investigación y uso en el ámbito gerencial, por ejemplo, en lo referido a fijación de objetivos y medición de su cumplimiento. Igualmente, se evidenciaron convergencias entre algunas de las perspectivas teóricas revisadas respecto al valor como métrica de la eficacia de la ejecución de la estrategia y de la contribución de la organización a la satisfacción de expectativas de sus grupos de interés. Este artículo identifica convergencias y divergencias en las escuelas más representativas de la administración estratégica, lo que permite formular de manera original una definición integral de desempeño, un modelo conceptual integrador en el cual el valor se constituye en métrica eficaz del desempeño empresarial.

Palabras clave: desempeño, performance, administración. estratégica, IO, RBV, DC, KBV.

\begin{abstract}
The purpose of this paper is to contribute with a comprehensive understanding of firm-performance on strategic management literature, conceptualizing its impact from a multi-level and multidimensional perspective. Likewise, the value is proposed as a metric of the firm- performance in three different levels of action. The definition and meaning of performance are examined through a literature review, including seminal - theoretical and empirical papers. The literature review comprises Industrial Organization (IO), Resources Based-View (RBV), Knowledge Based-View (KBV) and Dynamic Capabilities (DC) as essential theoretical perspectives in the Strategic Management area of study. Similarities and differences between stances are established. The results are presented in an original integrative conceptual model. In Strategic Management (SM) the expression 'firm performance' (FP) has been used to explain the strategy execution results. However, the literature review exposes performance as a construct that has suffered ambiguity in

a Universidad Externado de Colombia, Bogotá, Colombia. Tecnológico de Monterrey, Ciudad de México, México. Correo electrónico: ochaconf@tec.mx

b EGADE Business School. Tecnológico de Monterrey, Monterrey, México. Correo electrónico: laura.zapata@tec.mx
\end{abstract}


its definition and lack of epistemological delimitation. These limitations show performance as an outcome, difficult to understand, research, and apply in a managerial context. The present study identifies the convergences and divergences that the performance concept presents in the most representative schools of Strategic Management. A comprehensive definition of performance and a conceptual integrative model in which value is proposed as integrative metric of business performance.

Keywords: Performance, Strategic Management, IO, RBV, DC, KBV.

\section{Introducción}

La estrategia empresarial no es exclusiva de grandes empresas en entornos estables. La estrategia tiene como objetivo primordial responder a las necesidades y a las preferencias del mercado, a los cambios del entorno, al tiempo que permite asegurar un propósito común, alcanzar las metas propuestas mediante planes de acción, es decir, una forma de gestión definida y con expectativas de resultado claras.

En el actual entorno de negocios, caracterizado por su gran complejidad e incertidumbre, se requiere que las organizaciones, sin importar su tamaño o giro de negocio, formulen y ejecuten estrategias oportunas, claras y flexibles para asegurar el cumplimiento de sus objetivos. En este medio cambiante, las organizaciones necesitan monitorear constantemente sus procesos y resultados. Respecto a los procesos, se requiere comprender cómo lo están haciendo (desempeño) y, en relación con los resultados, se necesita identificar cuál es la brecha entre lo obtenido y lo esperado (medición del desempeño), de manera que puedan emprender acciones para lograr los objetivos. Este proceso sistemático de revisión de la estrategia da origen a la medición del desempeño empresarial, que tiene como fin valorar integralmente la forma como se comporta la organización frente a sus objetivos, procesos y resultados.

El desempeño superior es la efectividad esperada por las empresas a partir de la ejecución de sus estrategias. Su importancia para la administración estratégica radica, según Venkatraman y Ramanujam (1986), en tres razones fundamentales: teórica, empírica y gerencial. Teóricamente, el desempeño es un concepto central en el estudio de la estrategia: "Most strategic management theories either implicitly or explicitly underscore performance implications, since performance is the time test of any strategy" (p. 802). Lo anterior alude a la relación que tiene el desempeño con preguntas esenciales de la estrategia como, por ejemplo, ¿por qué las firmas tienen diferente desempeño? (Rumelt, Schendel y Teece, 1991).

Entre tanto, en lo empírico, el constructo desempeño se ha utilizado de manera muy extendida para examinar el contexto y los procesos de la estrategia (Venkatraman y Ramanujam,1986). Es así como más de dieciocho mil artículos publicados en el campo del management, desde 1980 a la fecha, están relacionados con este tema (WOS, 2020). Finalmente, en el nivel gerencial, la comprensión de desempeño se da en varios ámbitos, especialmente en lo relativo a los procesos, notable en el interés por medir tiempos, costos e incidencias relacionadas con atributos tales como la calidad, la eficiencia, la productividad, entre otros. En este nivel, es evidente el interés por comprender el desempeño en el ámbito de los resultados para observar si se ha cumplido el objetivo, si se han obtenido mejoras en resultados respecto a vigencias anteriores o, sencillamente, para comparar los logros respecto a otros competidores de la industria.

El desempeño superior es la variable dependiente utilizada con mayor frecuencia por los autores de las escuelas más representativas de la administración estratégica (March y Sutton, 1997; Newbert, 2008), por lo mismo, se hace imperante resolver qué significa desempeño superior, ya que, con ello, se resolvería un problema de delimitación epistemológica observable en la teoría. Esto significa que la variable "desempeño" en el campo de la estrategia carece de unidad de análisis. Fenómeno que se puede explicar a partir del tratamiento poco diferenciado hecho al referirse indistintamente al desempeño de los individuos en la organización y al desempeño de la organización como un todo, o en la falta de unidad de medida (cuál es la métrica apropiada para operacionalizar el desempeño superior de la firma), por ejemplo, en la visión de utilidades o rentas como métricas del desempeño, lo que configura ambigüedad en el tratamiento del concepto (Peteraf, 1994; Bowman y Ambrosini, 2000).

En atención a esta problemática, mediante un análisis del concepto "desempeño superior" en las principales perspectivas de la administración estratégica, el presente artículo expone las convergencias y las divergencias más significativas de este concepto. Mediante una revisión sistemática de la literatura, se identificaron 41 artículos, varios de ellos seminales, entre más de 900 publicaciones en el campo de la administración estratégica relacionadas con performance o firm performance.

Como contribución, se presentan una definición de desempeño de la empresa y un modelo conceptual 
integrador del desempeño empresarial. Este modelo permite superar algunos de los problemas de conceptualización, delimitación y medición del desempeño empresarial antes mencionados, facilitando investigaciones posteriores sobre el desempeño de la firma en el campo de la estrategia empresarial. Asimismo, se enfatiza en la medición del valor como métrica del desempeño de la empresa, aspecto que será atendido mediante la validación y la contrastación del modelo integrativo conceptual propuesto en futuras investigaciones.

\section{Revisión de literatura}

La revisión de literatura fue el método de investigación seleccionado para consolidar una base de artículos relevantes, de contrastada calidad, relacionados con la pregunta de investigación y ceñidos a las cuatro perspectivas teóricas de administración estratégica previamente seleccionadas. Las etapas en las que se surtió la revisión de literatura fueron formulación de pregunta de investigación (¿qué es desempeño superior en administración estratégica?), búsqueda, selección y evaluación de los artículos, análisis de contenido de los artículos seleccionados y descripción de los resultados (Gusmão et al., 2018).

Para la búsqueda y selección de los artículos se incluyeron publicaciones de la base de datos Scopus en el periodo 1990-2020. De los artículos localizados en este periodo, se identificaron en el título, resumen y palabras clave: performance, firm-performance, Resources Based-view, competitive advantage, Knowledge Basedview, Dynamic capabilities, y se obtuvieron 27 artículos. A fin de robustecer la base de artículos por analizar, se agregaron las siguientes revistas: Academy of Management Review, Strategic Management Journal, Harvard Business Review, MIT Sloan Review of Management con lo cual se identificaron 19 documentos adicionales. Se excluyeron cinco publicaciones no afines a administración estratégica y conferencias (proceedings) $(\mathrm{N}=41)$. En el apéndice, se presenta la tabla A1 que discrimina los autores, publicaciones, preguntas de investigación y contribución de los artículos seleccionados para el estudio.

Del análisis del contenido, se puede argumentar que autores de la administración, específicamente de la administración estratégica, utilizan en sus disertaciones diferentes denominaciones para el desempeño (Richard et al, 2009 ): ventaja competitiva y utilidad (Porter, 1985; Grant, 1996; Barney, 1991), rentas (Penrose, 1959; Mahoney y Pandian, 1992); Amit y Schoemaker, 1993; Peteraf, 1993,1994; Peteraf y Barney, 2003), retorno (Wernerfelt, 1984; Coff, 1999), valor económico (Bowman y Ambrosini, 2000; Peteraf y Barney, 2003) e incluso acuden a referencias abstractas como Winter (1995):
"The idea of superior financial performance may be evoked by a range of phrases such as 'above normal returns', 'high quasi-rents', 'value-creation' and other near-synonyms for 'making money'" (p. 168).

En la literatura empírica es igualmente evidente el problema de unidad de medida. Richard et al. (2009), actualizando las apreciaciones hechas por March y Sutton (1997), efectuaron una revisión de la literatura en las siguientes revistas: Strategic Management Journal, Academy of Management Journal, Administrative Science Quarterly, Journal of International Business Studies y Journal of Management. Estos autores encontraron que el $29 \%$ de los 213 artículos publicados por estas revistas en el periodo 1993-2000 versaban sobre desempeño como variable dependiente, independiente o de control, y empleaban 207 diferentes medidas de desempeño(Richard et al., 2009). Bien precisan Bititci et al. (2018) y Smith y Bititci (2017) que las nociones de performance (efectividad de una acción), performance measurement (qué medir) y performance management (cómo aprovechar las medidas para manejar el desempeño de la empresa) no siempre son tan claras en la literatura.

Por lo anterior, se hace manifiesta la necesidad de contribuir a la definición del concepto desempeño, dada su importancia para la estrategia en los ámbitos académico y empresarial. En este sentido, en la siguiente sección, se abordan cuatro perspectivas teóricas de la administración estratégica con diferentes posturas frente al desempeño, que servirán de fundamento al modelo conceptual propuesto en el presente estudio.

\section{Desempeño superior desde la perspectiva}

de organización industrial

El desempeño en la perspectiva teórica organización industrial es comprendido como un resultado, producto de la consolidación de la ventaja competitiva. Porter (1981, 1991), basado en trabajos previos de Mason (1952), Bain (1956, 1968, 1972) y Andrews (1971), identifica el "éxito de la firma" como una consecuencia de la posición competitiva, que conlleva a un desempeño financiero superior, y se ve influenciado por las condiciones de la industria en la que la empresa opera.

Para Porter (1991), son necesarias tres condiciones para el "éxito de la firma" en la perspectiva de organización industrial: un conjunto de objetivos y políticas funcionales (estrategia) que definan su posicionamiento estratégico, el alineamiento de esta estrategia de posicionamiento con las fortalezas y debilidades de la firma, en función de oportunidades y amenazas externas; y el aprovechamiento de las competencias distintivas que soporten la ventaja competitiva. 
La firma, argumenta Porter (1991), es una colección de actividades económicas discretas e interrelacionadas, en donde es precisamente la actividad la unidad básica fuente de ventaja competitiva. Para Porter (1991), realizar una actividad o grupo de actividades, consolida unos activos en forma de habilidades, rutinas organizacionales y conocimiento. Así, en la perspectiva de la organización industrial, el desempeño superior se asimila a la consecución de ventaja competitiva, especialmente a través de la ejecución coherente de la estrategia de posicionamiento estratégico.

\section{Desempeño superior desde la perspectiva}

\section{de recursos y capacidades}

Desde la perspectiva teórica de los recursos y capacidades, la heterogeneidad de los recursos y la configuración organizacional son las dos condiciones necesarias identificadas como causas del desempeño superior (Barney, 1991), el cual se asimila a la consecución y el mantenimiento de la ventaja competitiva (Newbert, 2008). La heterogeneidad de recursos se manifiesta en la posesión de recursos y capacidades con características VRIN: valiosos, raros, inimitables y no sustituibles (Barney, 1991). Sin embargo, no basta con la posesión de recursos y capacidades VRIN para garantizar la consolidación de un desempeño superior (Mahoney y Pandian, 1992). Por tanto, la configuración organizacional para aprovechar, explotar o capitalizar estos recursos se hace fundamental (Penrose, 1959; Teece, Pisano y Shuen, 1997; Barney, 1991; Eisenhardt y Santos, 2000; Moliterno y Wiersema, 2007).

Como alternativa a la comprensión de la ventaja competitiva como sinónimo de desempeño superior, Peteraf $(1993,1994)$ propone que el desempeño superior de las empresas está relacionado con la generación de rentas e identifica diez diferentes tipos, por lo que precisa el imperativo de producir rentas ricardianas - derivadas de la diferenciación de los recursos - y cuasi-rentas - retornos que exceden el costo de oportunidad a corto plazo de un factor-. Posteriormente, Peteraf y Barney (2003) exponen una definición de desempeño superior que da un giro importante a la comprensión del desempeño basado en rentas hacia la teoría del valor: "The capacity to create more economic value - an additive combination of producer and consumer surplus - than the marginal competitor in its product market" (p. 314).

\section{Desempeño superior desde la perspectiva}

de capacidades dinámicas

Desde capacidades dinámicas, enfoque filial de recursos y capacidades (Helfat y Peteraf, 2003, Einsenhart y Martin, 2000), Barreto (2010), y Ambrosini y Bowman (2009) argumentan que existen tres aproximaciones diferentes al desempeño en la literatura de estrategia. En primera instancia, observan el desempeño de la firma en función directa de las capacidades dinámicas, que son habilidades de las firmas para integrar, construir y reconfigurar las competencias internas y externas en función del rápido cambio en el entorno (Teece, Pisano, y Shuen, 1997). Desde esta perspectiva, las capacidades dinámicas de las firmas explican el éxito o fracaso, la ventaja competitiva o la creación de riqueza (Barreto, 2010).

En la segunda aproximación, se afirma que la relación entre capacidades dinámicas y desempeño está sujeta a acciones de identificación y explotación de las oportunidades. Es así como para Eisenhardt y Martin (2000) la ventaja competitiva a largo plazo no depende de las capacidades dinámicas por sí mismas, sino de las configuraciones de recursos creadas por las capacidades dinámicas y en un uso más oportuno, inteligente y favorable que la competencia (Eisenhardt y Martin, 2000).

Por último, en la tercera aproximación, se sustenta que la relación entre las capacidades dinámicas y el desempeño superior es indirecta. Así, Zott (2003) argumenta que las capacidades dinámicas pueden influenciar el desempeño mediante modificaciones de la configuración de recursos o rutinas. Aun cuando las firmas puedan contar con idénticas capacidades dinámicas, podrían configurar de manera diferente sus recursos y, con ello, obtener niveles de desempeño diferente (Barreto, 2010).

\section{Desempeño superior desde la perspectiva} basada en el conocimiento

En la medida en que las capacidades dinámicas cobraron valor como activos, especialmente aquellos intangibles que son aprovechados para lograr sus objetivos, evolucionaron enfoques alternativos al enfoque de recursos y capacidades, cuya atención se concentró en estudiar la forma como las actividades organizacionales se tornaban más dinámicas, para luego constituirse en prácticas características o rutinas (Nelson y Winter, 2002) y, posteriormente, en conocimiento. Con la progresión de las actividades a rutinas y luego a conocimiento, se reconoció su capacidad de generar ventajas, y, por tanto, ser portadoras de valor, esto es, cuanto más contribuyen estas actividades, rutinas y conocimientos a la generación y la explotación de ventajas, más valiosas son.

A partir de esta comprensión surgió la perspectiva basada en el conocimiento, enfoque que considera el conocimiento como la fuente de ventaja competitiva y del desempeño superior (Grant, 1996, 1997; Eisenhardt y Santos, 2000). El conocimiento está en los individuos, 
embebido en los principios, las políticas y los procesos de las organizaciones. Este conocimiento evoluciona mediante path dependencies, replicación y recombinación del conocimiento existente. Según Nonaka, Toyama y Konno (2000) esta evolución sigue un patrón de espiral, altamente iterativo, mediante la acumulación y flujo continuo (Dierickx, Cool y Constance, 1989) entre conocimiento tácito y explícito, individual y organizacional (Eisenhardt y Santos, 2000).

La tabla 1 presenta una síntesis de las categorías de análisis a partir de las cuales se revisaron las perspectivas teóricas anteriores e introduce el análisis de convergencias y divergencias que se desarrolla en la discusión a continuación.

\section{Discusión}

Las perspectivas de organización industrial, recursos y capacidades, capacidades dinámicas y basadas en el conocimiento sustentan sus respectivos argumentos en los que se pueden identificar similitudes y diferencias. Del lado de las similitudes, se observa la dinámica de una firma en relación con un entorno competitivo o industria. A partir de estas similitudes se construye el modelo integrador, expuesto en la figura 1, y la forma como las empresas reconocen su entorno, caracterizado por dinámicas de cambio económico, político, social y tecnológico que se traducen en oportunidades o amenazas que deben ser identificadas y capitalizadas (Porter, 1991; Teece et al., 1997). A la par, reconocen la existencia de condiciones internas que podrían potenciar la captura de oportunidades (March, 1991) o limitar su aprovechamiento. Este conjunto de condiciones se denomina en este estudio como el nivel de alineamiento estratégico.

Igualmente, estas mismas perspectivas coinciden en reconocer la existencia de una configuración singular de recursos y capacidades —en adelante el nivel organizacional-, cuyo provecho sustenta el eventual acceso y, en dado caso, mantenimiento de la ventaja competitiva; aunque es notable que los autores de las perspectivas analizadas le confieren denominaciones distintas (v. gr. actividades, rutinas, capacidades de absorción y capacidades dinámicas).

Más allá de la denominación de los componentes del nivel organizacional, las diferencias más notables entre las perspectivas teóricas analizadas radican en cómo estas comprenden el desempeño. En la perspectiva de organización industrial, se asevera que la rentabilidad de la firma depende de dos elementos: posición competitiva y efectos de la industria (Porter, 1991). En tanto, en recursos y capacidades, las rentas provienen de la capacidad de explotar los recursos y capacidades valiosos, raros, inimitables e insustituibles y, si estos recursos y capacidades han sido adquiridos o desarrollados (Barney, 1991). Mientras que en la perspectiva basada en el conocimiento, las rentas provienen de la consolidación de la ventaja competitiva a partir del conocimiento (Grant, 1996, 1997).

Una razón fundamental que explica las diferencias de enfoque entre estas perspectivas de estrategia

Tabla 1. Síntesis de posturas teóricas sobre desempeño

\begin{tabular}{|c|c|c|c|c|c|}
\hline Perspectiva & Influencia & Principales Autores & Noción de desempeño & $\begin{array}{l}\text { Desempeño } \\
\text { depende de: }\end{array}$ & $\begin{array}{l}\text { Formas de medición } \\
\text { del desempeño }\end{array}$ \\
\hline $\begin{array}{l}\text { Organización } \\
\text { industrial }\end{array}$ & \multirow{3}{*}{$\begin{array}{l}\text { Teoría } \\
\text { económica } \\
\text { clásica }\end{array}$} & $\begin{array}{l}\text { Andrews, K. (1971) } \\
\text { Mason, E. (1952) } \\
\text { Bain, J. (1956) } \\
\text { Porter, M. (1981, 1981) }\end{array}$ & $\begin{array}{l}\text { - Éxito } \\
\text { - Ventaja competitiva } \\
\text { - Desempeño superior } \\
\text { - Posicionamiento } \\
\text { - estratégico }\end{array}$ & $\begin{array}{l}\text { Posicionamiento } \\
\text { y efectividad } \\
\text { industrial }\end{array}$ & $\begin{array}{l}\text { - Utilidad } \\
\text { - Rentabilidad } \\
\text { - Participación Mercado }\end{array}$ \\
\hline $\begin{array}{l}\text { Recursos y } \\
\text { capacidades }\end{array}$ & & $\begin{array}{l}\text { Barney, J. (1991) } \\
\text { Peteraf, M. (1993,1994) } \\
\text { Mahoney, J. (1992) } \\
\text { Teece, D. (1997) } \\
\text { Wernerfelt, B. (1984) }\end{array}$ & $\begin{array}{l}\text { - VRIN } \\
\text { - Ventaja competitiva }\end{array}$ & \multirow{2}{*}{$\begin{array}{l}\text { Capacidad de } \\
\text { explotar recursos } \\
\text { y capacidades } \\
\text { VRIN (adquiridas o } \\
\text { desarrolladas) }\end{array}$} & $\begin{array}{l}\text { - Utilidad económica } \\
\text { - Rentabilidad } \\
\text { - Valor }\end{array}$ \\
\hline $\begin{array}{l}\text { Recursos y } \\
\text { capacidades } \\
\text { dinámicas }\end{array}$ & & $\begin{array}{l}\text { Ambrosini, V. (2009) } \\
\text { Bowman, C. (2009) } \\
\text { Eisenhart, K. (2000) } \\
\text { Helfat, C. (2003) } \\
\text { Martin, J. (2000) } \\
\text { Zott, C. (2003) }\end{array}$ & $\begin{array}{l}\text { - Capacidades dinámicas } \\
\text { - Rentas ricardianas y } \\
\text { monopolísticas }\end{array}$ & & $\begin{array}{l}\text { - Rentabilidad } \\
\text { - Valor }\end{array}$ \\
\hline $\begin{array}{l}\text { Basada en } \\
\text { Conocimiento }\end{array}$ & $\begin{array}{c}\text { Teoría } \\
\text { evolucionista }\end{array}$ & $\begin{array}{l}\text { Dierickx, I. (1989) } \\
\text { Eisenhart, K. (2000) } \\
\text { Grant, R. (1996) } \\
\text { Nelson, R. (2002) } \\
\text { Nonaka, I. (2000) } \\
\text { Winter, S. (2002) }\end{array}$ & $\begin{array}{l}\text { - Capacidades absorbentes } \\
\text { - Conocimiento como } \\
\text { ventaja competitiva } \\
\text { - Rentas shumpeterianas }\end{array}$ & $\begin{array}{l}\text { Consolidación } \\
\text { de ventajas } \\
\text { competitivas a partir } \\
\text { del conocimiento }\end{array}$ & $\begin{array}{l}\text { - Valor } \\
\text { - Activos } \\
\text { de conocimiento }\end{array}$ \\
\hline
\end{tabular}


es la influencia de la teoría económica. La teoría de la ventaja competitiva porteriana y la de recursos y capacidades están notablemente influenciadas por la teoría del equilibrio económico (Solow, 1956), que centra su atención en el paradigma de la maximización de las utilidades (Nelson y Winter, 2002); mientras que la perspectiva basada en el conocimiento ha recibido el influjo de la teoría evolucionista, cuyas preguntas fundamentales influyeron en la teoría de la competencia y la teoría del cambio tecnológico e institucional (Nelson y Winter, 2002).

En línea con lo anterior, la teoría económica influye también en la manera como las perspectivas en contrastación comprenden las rentas referidas al desempeño. En organización industrial y en recursos y capacidades, el desempeño de la firma está asociado con el fenómeno de la generación de rentas sin observar su apropiación (Peteraf, 1993; Barney, 1991), mientras en la perspectiva basada en conocimiento se percibe la apropiación desde la perspectiva de los grupos de interés, es decir, la ventaja competitiva no conduce por sí misma a niveles de desempeño superior; ello depende de cuan apropiadas sean las rentas por parte de los grupos de interés (Coff, 1999). Además, la heterogeneidad de recursos principio de organización industrial y recursos y capacidades - genera rentas ricardianas y monopolísticas
(Peteraf, 1993, p. 185), mientras la perspectiva basada en conocimiento advierte la posibilidad de generar rentas shumpeterianas, es decir, aquellas que se fundamentan en la innovación en atención a regímenes de rápidos cambios en el entorno (Ambrosini y Bowman, 2009).

A continuación, a manera de síntesis y como contribución fundamental, se presenta un modelo conceptual que integra las dimensiones comprendidas por las diferentes perspectivas teóricas de administración estratégica y comprende en tres niveles de análisis la noción del desempeño de la firma.

El modelo conceptual expuesto en la figura 1 comprende el desempeño como un fenómeno multidimensional mediante los niveles estratégico, organizacional y de resultados. El nivel estratégico representa el proceso racional y continuo mediante el cual la firma efectúa el análisis del entorno competitivo y precisa las acciones estratégicas: actividades (Porter, 1981) y rutinas (Nelson y Winter, 2002; Eisenhart y Martin, 2000) que habrá de ejecutar haciendo uso de sus recursos y capacidades (Barney, 1991). Estos se encuentran en el nivel organizacional, que es en donde se configuran, explotan (March, 1991) y aprovechan. Esta dinámica es iterativa y permite a la organización capitalizar experiencias que pueden modificar la configuración de recursos o capacidades,

Figura 1. Modelo conceptual de desempeño empresarial

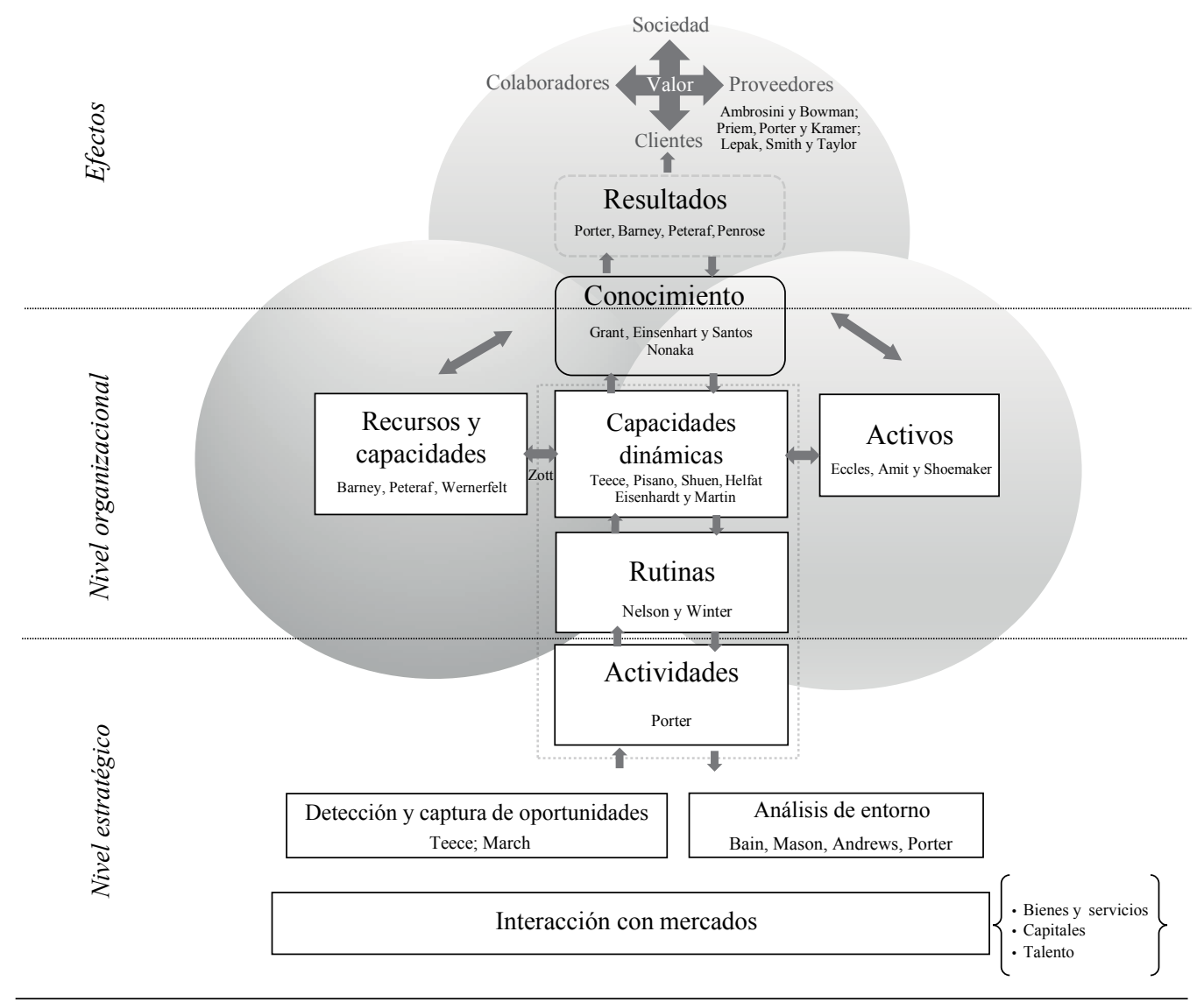


con lo cual la empresa logra que estos sean más valiosos, en relación con su importancia estratégica para la firma (como fuente de ventaja), y también con respecto a su capacidad de producir rentas (Peteraf y Barney, 2003).

El nivel de resultado comprende las rentas que, según la revisión de literatura, atienden las expectativas de productividad en el corto plazo y de retorno en el largo. Cabe aclarar que no todas las rentas son susceptibles de ser distribuidas, pues algunas se encuentran representadas en activos estratégicos, por tanto, no enajenables. De esta manera, la empresa genera valor (Bowman y Ambrosini, 2000), distribuye o comparte con sus grupos de interés principales: clientes (Priem, 2007; Smith y Colgate, 2007; Möller y Törrönen, 2003; Porter y Kramer, 2011); y otra parte del valor generado es retenido por la organización (Lepak, Smith, y Taylor, 2007) en forma de reconocimiento social y relaciones clave de negocio, entre otros intangibles.

¿Es posible conciliar la noción de desempeño de las perspectivas teóricas en la administración estratégica? A instancias del modelo propuesto, el desempeño de una empresa puede ser definido como un constructo multidimensional, comprendido por los niveles estratégico, organizacional y de resultado; cuya dinámica explica la efectividad de la empresa al interactuar con los mercados (de bienes y servicios, de capitales y de trabajo), identificar oportunidades y amenazas, establecer propósitos y objetivos, definir actividades o rutinas, hacer uso de recursos y capacidades para lograr la ejecución sistemática de propósitos, políticas y procesos, y obtener resultados que satisfacen las expectativas de la organización, relacionadas con el cumplimiento de los objetivos, y las de los grupos de interés, relacionadas con la satisfacción de necesidades (clientes), rentabilidad (inversionistas), compromiso e involucramiento (colaboradores), sustentabilidad (sociedad), entre otros. Es decir, el desempeño - comprendido en los tres niveles de análisis explicados anteriormente- es observable en la forma como la firma se relaciona con el mercado, y en cómo define y ejecuta su estrategia, realiza sus procesos, gestiona recursos $\mathrm{y}$, adicionalmente, - no solamente, como ha sido visto tradicionalmente en la teoría - en los resultados que obtiene.

Respecto a la medición del desempeño de la empresa, el modelo propone acudir a la noción del valor, concepto que no está representado únicamente en la rentabilidad marginal del capital (enfoque económico y financiero), pues el valor está embebido, además, en los recursos VRIN y en las capacidades distintivas que sustentan la ventaja competitiva (Amit y Schoemaker, 1993), en la retribución que entrega a sus grupos de interés y en el conocimiento que la firma genera, explota y acumula. Esto significa que el valor económico que las empresas producen no subyace solo en el resultado que obtienen o en las retribuciones, compensaciones y contribuciones que hacen a sus grupos de interés; sino que está representado también en los recursos estratégicos que permanecen en la firma, incluido el conocimiento.

El valor económico, en términos de métrica del desempeño, tiene como ventajas exceder el corto plazo - que caracteriza a los indicadores contables como la utilidad-, permitir la medición objetiva de los resultados actuales y futuros de la firma, e incluso, en contraposición a postulados de la perspectiva de recursos y capacidades, que considera que los activos estratégicos no son enajenables (Bowman y Ambrosini, 2000), hace posible la valoración de activos estratégicos en situaciones particulares de mercado como, por ejemplo, fusiones, adquisiciones, alianzas o cesión de franquicias.

Además, el valor tiene arraigo en la teoría económica, lo cual facilita su formalización. Aún más, existen diversos modelos de medición financiera aplicados a la determinación del valor de los activos, entre ellos, método de flujo de caja descontado, opciones reales, Black y Sholes, costo promedio ponderado de capital, entre otros, lo cual permitiría avanzar en la operacionalización y la validación empírica. Sin embargo, existen tensiones por resolver en el campo teórico entre defensores de la maximización del valor a favor del accionista como único objetivo (Jensen, 2001; Copeland y Dolgoff, 2006 ), y posturas teóricas, tan contemporáneas como divergentes, como, por ejemplo, el valor compartido (Porter y Kramer, 2011), Stakeholders model (Atkinson, Waterhouse y Wells, 1997); la sustentabilidad (Ameer y Othman, 2012; Gupta y Kumar, 2013), The Triple Bottom line (Elkington, 1997; Norman y Macdonald, 2004), entre otras, que impulsan - con razón - a las empresas y a la academia a revisar lo que significa la medición del desempeño superior de la firma en relación con su real impacto en la sociedad, más allá de la medición del retorno sobre la inversión o la productividad de los recursos.

\section{Conclusiones}

El presente estudio expone las falencias que en la definición del constructo "desempeño" generan ambigüedad y, por tanto, tienden a dificultar su operacionalización, análisis y aplicabilidad en el ámbito gerencial. El desempeño no es solamente la utilidad o la renta marginal producida en un periodo de tiempo, quizás sean estas sus aproximaciones más visibles. No obstante, el modelo conceptual propuesto precisa que el desempeño es un fenómeno multidimensional, que parte de la forma como la empresa interactúa con el mercado, no solo desde la perspectiva de oferta y demanda de bienes y servicios, capital y talento; sino también desde la perspectiva en la 
cual la empresa captura y capitaliza (o no) las oportunidades subyacentes mediante la afirmación intencional de propósitos convertidos en estrategias de negocio.

De igual forma, el modelo precisa que la empresa configura un arreglo de características organizacionales, tales como políticas, procesos, recursos y capacidades, cultura, que dan sentido y carácter a la manera en la que la firma se comporta en función de sus objetivos, y define su desempeño. Finalmente, en el resultado, el modelo propuesto reconoce el valor generado, retenido y compartido de la empresa a favor de todos los grupos de interés.

El modelo integral permite a las organizaciones contar con una visión integral de su impacto interno en sus diferentes niveles, y externo con respecto a sus diferentes grupos de interés. Plantea que no solo es posible medir el desempeño empresarial como una función económica, sino también identificar este valor intrínseco y compartido con la sociedad. Asimismo, el modelo es una primera aproximación que permite a las organizaciones planear y ejecutar su estrategia con base en mediciones integrales, multidimensionales y multinivel.

Queda claro que la estrategia va más allá de la participación de la alta gerencia y el consejo de la empresa y que cada uno de los integrantes, los procesos y las actividades de la organización tienen un papel clave en la consecución de los objetivos y la consolidación del valor de los activos estratégicos de la empresa. Si bien es cierto que la medición del desempeño ofrece la oportunidad de contar con información precisa sobre la diferencia entre lo obtenido y lo esperado, de manera que puedan emprenderse ajustes o reconducción de acciones para lograr los objetivos planteados, en el entorno actual se vuelve relevante medir también el valor e impacto que generan las empresas en sus grupos de interés externos, esto es, en la sociedad.

Una futura investigación permitirá contrastar empíricamente el modelo conceptual propuesto. En una primera fase, una investigación cualitativa ofrecerá la oportunidad de robustecer los conceptos, validar y expandir las relaciones que lo integran. Posteriormente, en una segunda fase, una investigación cuantitativa ofrecerá una perspectiva más profunda de identificación de indicadores integrales y multidimensionales de medición del desempeño, fundamentados en el valor.

Si bien la noción de valor como resultado e indicador del desempeño es robusta, teórica y empíricamente, sigue siendo necesario un debate y una revisión adicional, no solo desde las perspectivas teóricas aquí expuestas, sino ampliada y confrontada desde otros enfoques como el valor compartido, la sostenibilidad, la responsabilidad social, cuyo análisis es fundamental para la comprensión amplia y exhaustiva del sentido de propósito de las empresas, el impacto de la estrategia empresarial en el desempeño superior de las firmas y de estas en la sociedad.

\section{Referencias}

Ambrosini, V., \& Bowman, C. (2009). What are dynamic capabilities and are they a useful construct in strategic management? International Journal of Management Reviews, 11(1), 29-49.

Ameer, R., \& Othman, R. (2012). Sustainability practices and corporate financial performance: A study based on the top global corporations. Journal of Business Ethics, 108(1), 61-79. https://doi.org/10.1007/s10551011-1063-y

Amit, R., \& Schoemaker, P. (1993). Strategic assets and organizational rent. Strategic Management Journal, 14(1), 33-46.

Andrews, K. (1971). The concept of corporate strategy. Dow Jones-Irwin.

Atkinson, A., Waterhouse, J., \& Wells, R. (1997). A stakeholder approach to strategic performance measurement. Sloan Management Review, 38(3), 25-38.

Bain, J. (1956). Barriers to new competition. Harvard University Press.

Bain, J. (1968). Industrial organization (2 ed.).Wiley.

Bain, J. (1972). Essays on proce theory and industrial organization. Little, Brown.

Barney, J. (1991). Firm resources and sustained competitive advantage. Journal of Management, 17(1), 99-120. https://doi.org/10.1177/014920639101700108

Barreto, I. (2010). Dynamic capabilities: A review of past research and an agenda for the future. Journal of Management, 36(1), 256-280. https://doi. org/10.1177/0149206309350776

Bititci, U., Bourne, M., Farris Cross, J., Nudurupati, S., \& Sang, K. (2018). Towards a theoretical foundation for performance measurement and management. International Journal of Management Reviews, 20(3), 653660. https://doi.org/10.1111/ijmr.12185

Bowman, C., \& Ambrosini, V. (2000). Value creation versus value capture: Towards a coherent definition of value in strategy. British Journal of Management, 11(1), 1-15. https://doi.org/10.1111/1467-8551.00147

Coff, R. (1999). When competitive advantage doesn't lead to performance: The resource-based view and stakeholder bargaining power. Organization Science, 10(2), 119. https://doi.org/10.1287/orsc.10.2.119

Copeland, T., \& Dolgoff, A. (2006). Expectations-Based management. Journal of Applied Corporate Finance, 18(2), 82-98. https://doi.org/10.1007/978-1-46149173-6

Dierickx, I., Cool, K., \& Constance, B. (1989). Asset stock accumulation and sustainability of competitive advantage. Management Science, 35(12), 1504-1511. https://doi.org/10.1287/mnsc.35.12.1504 
Eisenhardt, K., \& Santos, F. (2000). Knowledge-based view: A new theory of strategy. In Handbook of Strategy and Management. Sage Publications.

Eisenhardt, K., \& Martin, J. (2000). Dynamic capabilities: what are they? Strategic Management Journal. https:// doi.org/10.1002/1097-0266(200010/11)21:10/11<1105: AID-SMJ133>3.0.CO ;2-E

Elkington, J. (1997). Cannibals with forks: The triple bottom lineof 21st century business. Capstone Paperback.

Grant, R. (1996). Towards a knowledge-based theory of the frim. Strategic Management Journal, 109-122. https://doi.org/10.2307/2486994

Grant, R. (1997). The knowledge-based view of the firm: implications for management practice. Long Range Planning Journal, 30(3), 450-454. https://doi. org/10.1016/S0024-6301(97)00025-3

Gupta, S., \& Kumar, V. (2013). Sustainability as corporate culture of a brand for superior performance. Journal of World Business, 48(3). https://doi.org/10.1016/j. jwb.2012.07.015

Gusmão, R., Leal, W., Gonçalves, O., de Mattos, D., \& Veigas A., L. (2018). A literature-based review on potentials and constraints in the implementation of the sustainable development goals. Journal of Cleaner Production, 198, 1276-1288. https://doi.org/10.1016/j. jclepro.2018.07.102

Helfat, C., \& Peteraf, M. (2003). The dynamic resource-based view: Capability lifecycles. Strategic Management Journal, 24, 997-1010. https://doi. org/10.1002/smj.332

Jensen, M. (2001). Value maximization, stakeholder theory, and the corporate objective function. Journal of Applied Corporate Finance, 14(3), 8-21.

Lepak, D., Smith, K., \& Taylor, M. (2007). Value creation and value capture: A multilevel perspective. Academy of Management Review, 32(1), 180-194. https://doi. org/10.2307/20159287

Mahoney, J., \& Pandian, J. (1992). The resource-based view within the conversation of strategic management. Strategic Management Journal, 13(5), 363-380.

March, J. (1991). Exploration and exploitation in organizational learning. Organization Science, 2(1), 71- 87. https://doi.org/10.1287/orsc.2.1.71

March, J., \& Sutton, R. (1997). Organizational performance as a dependent variable. Organization Science, 8(6), 698-706.

Mason, E. (1939). Price and production policites of largescale enterprises. American Economic Review, 29, 61-74-
Moliterno, T., \& Wiersema, M. (2007). Firm performance, rent appropriation, and the strategic resource divestment capability. Strategic Management Journal, 28(11), 1065-1087. https://doi.org/10.1002/smj.630

Möller, K., \& Törrönen, P. (2003). Business suppliers value creation potential a capability-based analysis. Industrial Marketing Management, 32(2), 109-118. https://doi.org/10.1016/S0019-8501(02)00225-0

Nelson, R., \& Winter, S. (2002). Evolutionary theorizing in economics. Journal of Economic Perspectives, 16(2), 23-46. https://doi.org/10.1257/0895330027247

Newbert, S. (2008). Value, rareness, competitive advantage, and performance: A conceptual-level empirical investigation of the resource-based view of the firm. Strategic Management Journal, 29(7). https://doi. org/10.1002/smj.686

Nonaka, I., Toyama, R., \& Konno, N. (2000). SECI, $\mathrm{Ba}$ and leadership: A unified model of dynamic knowledge creation. Long Range Planning Journal, 33(1), 5-34. https://doi.org/10.1016/S00246301(99)00115-6

Norman, W., \& Macdonald, C. (2004). Getting to the Bottom of "Triple Bottom Line". Business Ethics Quarterly, 14(2), 243-262. https://doi.org/10.1016/j. ijpe.2012.01.03

Penrose, E. (1959). The theory of the growth of the firm (4th edition). Oxford University Press.

Peteraf, M. (1993). The cornerstones of competitive advantage: A resources-based view. Strategic Management Journal, 14(3), 179-191.

Peteraf, M. (1994). The two schools of thought in resource-based theory: Definitions and implications for research. Advances in Strategic Management, 10A, 153-158.

Peteraf, M., \& Barney, J. (2003). Unraveling the resource-based tangle. Managerial and Decision Economics, 24(4), 309-323. https://doi.org/10.1002/ mde. 1126

Porter, M. (1981). The contributions of industrial organization to strategic management. The Academy of Management Review, 6, 609-620. https://doi. org/10.2307/257639

Porter, M. (1985). Competitive advantage, creating and sustaining superior performance. The Free Press.

Porter, M. (1991). Towards a dynamic theory of strategy. Strategic Management Journal, 12(1), 95-117. https:// doi.org/10.1007/s13398-014-0173-7.2

Porter, M., \& Kramer, M. (2011). Creating shared value How to reinvent capitalism and unleash a wave of innovation and growth. Harvard Business Review, 39, 6277. https://doi.org/10.1108/09600039410055963 
Priem, R. (2007). A consumer perspective on value creation. Academy of Management Review, 32(1), 219235. https://doi.org/10.5465/AMR.2007.23464055

Richard, P., Devinney, T., Yip, G., \& Johnson, G. (2009). Measuring organizational performance: Towards methodological best practice. Journal of Management, 35(3), 718-804. https://doi.org/10.1177/01492063083 30560

Rumelt, R., Schendel, D., \& Teece, D. (1991). Strategic management and economics. Strategic Management Journal, 12, 5-29.

Smith, J., \& Colgate, M. (2007). Customer value creation: A practical framework. Journal of Marketing Theory and Practice, 15(1), 7-23. https://doi.org/10.2753/ MTP1069-6679150101

Smith, M., \& Bititci, U. (2017). Interplay between performance measurement and management, employee engagement and performance. International Journal of Operations and Production Management, 37(9), 12071228. https://doi.org/10.1108/IJOPM-06-2015-0313

Solow, R. (1956). A Contribution to the theory of economic growth. The Quarterly Journal of Economics, 70(1), 65-94. http://www.jstor.org/stable/1884513
Teece, D., Pisano, G., \& Shuen, A. (1997). Dynamic capabilties and strategic management. Strategic Management Journal, 18(7), 509-533. https://doi.org/10.1002/ (SICI) 1097-0266(199708)18:7<509::AID-SMJ882>3.0.CO;2-Z

Venkatraman, N., \& Ramanujam, V. (1986). Measurement of business performance in strategy research: A comparison of approaches. The Academy of Management Review, 11(4), 801-814. https://doi. org/10.2307/258398

Wernerfelt, B. (1984). A resource-based view of the firm. Strategic Management Journal, 5(2), 171- 180. https:// doi.org/10.1007/s10734-011-9485-0

Winter S. (1995). Four Rs of profitability: rents, resources, routines, and replication. In Resource-Based and Evolutionary Theories of the Firm, Montgomery CA (ed.). Kluwer: Boston, MA; 147-158. https://doi. org/10.1007/978-1-4615-2201-0_7.

Zott, C. (2003). Dynamic capabilities and the emergence of intraindustry differential firm performance: Insights from a simulation study. Strategic Management Journal, 24(2), 97-125. https://doi.org/10.1002/smj.288

\section{Apéndice}

Tabla A1. Cuadro resumen revisión de literatura

\begin{tabular}{|c|c|c|c|c|}
\hline Autor & Journal & Pregunta & Contribución & Citaciones (*) \\
\hline $\begin{array}{l}\text { Ambrosini, V. y Bowman, } \\
\text { C. }(2009)\end{array}$ & $\begin{array}{l}\text { International Journal of } \\
\text { Management Reviews }\end{array}$ & ¿Qué son capacidades dinámicas? & $\begin{array}{l}\text { Revisión y síntesis de literatura sobre capacidades } \\
\text { dinámicas. Identifica cómo se configuran a } \\
\text { partir de condiciones del ámbito y entorno } \\
\text { organizacional. Identifica el proceso de creación } \\
\text { de capacidades dinámicas. }\end{array}$ & 1917 \\
\hline $\begin{array}{l}\text { Ameer, R. y Othman, R. } \\
\text { (2012) }\end{array}$ & $\begin{array}{l}\text { Journal of Business } \\
\text { Ethics }\end{array}$ & $\begin{array}{l}\text { ¿Existe relación entre CSR y } \\
\text { desempeño? }\end{array}$ & $\begin{array}{l}\text { Identifica y valida relación bidireccional entre las } \\
\text { prácticas de responsabilidad social empresarial y } \\
\text { desempeño financiero. }\end{array}$ & 580 \\
\hline $\begin{array}{l}\text { Amit, R. y Schoemaker, } \\
\text { P. (1993) }\end{array}$ & $\begin{array}{l}\text { Strategic Management } \\
\text { Journal }\end{array}$ & $\begin{array}{l}\text { ¿Qué hace a las organizaciones } \\
\text { distintas y únicas? }\end{array}$ & $\begin{array}{l}\text { Precisa qué imperfeciones de mercado relacionadas } \\
\text { con los recursos y capacidades y las decisiones } \\
\text { discrecionales sobre asignación y desarrollo } \\
\text { producen diferencias en generación de rentas. }\end{array}$ & 14294 \\
\hline $\begin{array}{l}\text { Atkinson, A., Waterhouse, } \\
\text { J. y Wells, R. (1997) }\end{array}$ & $\begin{array}{l}\text { Sloan Management } \\
\text { Review }\end{array}$ & $\begin{array}{l}\text { ¿Cómo los grupos de interés } \\
\text { comprenden y evalúan el } \\
\text { desempeño de la empresa? }\end{array}$ & $\begin{array}{l}\text { Propone un modelo que reconoce la relación del } \\
\text { desempeño con objetivos primarios (relacionado } \\
\text { con expectativas de grupos de interés) y } \\
\text { secundarios (métricas para monitorear o mejorar } \\
\text { el desempeño). }\end{array}$ & ND \\
\hline Barney, J. (1991) & Journal of Management & $\begin{array}{l}\text { ¿Cuáles son las fuentes de } \\
\text { ventaja competitiva? }\end{array}$ & $\begin{array}{l}\text { Identifica cuatro indicadores empíricos del } \\
\text { potencial de una firma para generar ventajas } \\
\text { competitivas. }\end{array}$ & 23744 \\
\hline
\end{tabular}


Tabla A1. Cuadro resumen revisión de literatura.

\begin{tabular}{|c|c|c|c|c|}
\hline Autor & Journal & Pregunta & Contribución & Citaciones (*) \\
\hline $\begin{array}{l}\text { Bititci, U., Bourne, } \\
\text { M., Farris Cross, J., } \\
\text { Nudurupati, S. } \\
\text { y Sang, K. (2018) }\end{array}$ & $\begin{array}{l}\text { International } \\
\text { Journal of } \\
\text { Management } \\
\text { Review }\end{array}$ & $\begin{array}{l}\text { ¿Cuál es el nuevo contexto } \\
\text { de la medición } \\
\text { de desempeño? }\end{array}$ & $\begin{array}{l}\text { Identifica los retos que afronta la medición del desempeño desde una } \\
\text { perspectiva holística. }\end{array}$ & 13 \\
\hline Coff, R. (1999) & $\begin{array}{l}\text { Organization } \\
\text { Science }\end{array}$ & $\begin{array}{l}\text { ¿Qué recursos generan } \\
\text { renta y cómo son } \\
\text { apropiadas? }\end{array}$ & $\begin{array}{l}\text { Propone un modelo conceptual que explica desde pensamiento } \\
\text { sistémico cómo las rentas son observables a partir de métricas de } \\
\text { desempeño y son apropiadas por diferentes grupos de interés. }\end{array}$ & 615 \\
\hline $\begin{array}{l}\text { Dierickx, I. y } \\
\text { Cool, K. (1989) }\end{array}$ & $\begin{array}{l}\text { Management } \\
\text { Science }\end{array}$ & $\begin{array}{l}\text { ¿De qué depende } \\
\text { la sustentabilidad de las } \\
\text { ventajas competitivas? }\end{array}$ & $\begin{array}{l}\text { Desarrolla y argumenta conceptualmente sobre las condiciones } \\
\text { y configuraciones a partir de las cuales las empresas generan } \\
\text { determinados resultados a partir de la explotación, configuración y } \\
\text { acumulación de recursos y capacidades. }\end{array}$ & ND \\
\hline $\begin{array}{l}\text { Eisenhardt, K. y } \\
\text { Martin, J. (2000) }\end{array}$ & $\begin{array}{l}\text { Strategic } \\
\text { Management } \\
\text { Journal }\end{array}$ & $\begin{array}{l}\text { ¿Qué son las capacidades } \\
\text { dinámicas? }\end{array}$ & $\begin{array}{l}\text { Explica la noción de capacidades dinámicas desde la perspectiva } \\
\text { de recursos y capacidades, y sustenta que estas no son condición } \\
\text { suficiente para la ventaja competitiva. }\end{array}$ & 6803 \\
\hline Grant, R. (1996) & $\begin{array}{l}\text { Strategic } \\
\text { Management } \\
\text { Journal }\end{array}$ & $\begin{array}{l}\text { ¿Cuáles son los elementos } \\
\text { clave de la perspectiva } \\
\text { basada en el conocimiento? }\end{array}$ & $\begin{array}{l}\text { Explica la necesidad de coordinar el proceso de integración de } \\
\text { conocimiento. Identifica las diferencias entre la perspectiva basada } \\
\text { en el conocimiento y la teoría organizacional }\end{array}$ & 8200 \\
\hline $\begin{array}{l}\text { Helfat, C. y Peteraf, } \\
\text { M. (2003) }\end{array}$ & $\begin{array}{l}\text { Strategic } \\
\text { Management } \\
\text { Journal }\end{array}$ & $\begin{array}{l}\text { ¿Cómo evolucionan las } \\
\text { capacidades dinámicas a } \\
\text { lo largo del tiempo? }\end{array}$ & $\begin{array}{l}\text { Introduce el concepto de CLC (ciclo de capacidades) para evidenciar } \\
\text { la forma como se desarrollan y maduran las capacidades dinámicas } \\
\text { en una organización. }\end{array}$ & 1836 \\
\hline Jensen, M. (2001) & $\begin{array}{l}\text { Journal of } \\
\text { Applied } \\
\text { Corporate } \\
\text { Finance }\end{array}$ & $\begin{array}{l}\text { ¿Cuál es la función } \\
\text { objetivo de las empresas } \\
\text { en relación con los grupos } \\
\text { de interés? }\end{array}$ & $\begin{array}{l}\text { Sustenta que el beneficio social se maximiza cuando cada firma en la } \\
\text { economía maximiza su valor de mercado. Expone las condiciones de } \\
\text { mercado, así como las configuraciones de organización que promueven } \\
\text { dinámicas de maximización de la riqueza en el largo plazo. }\end{array}$ & 23510 \\
\hline $\begin{array}{l}\text { Lepak, D., Smith, K. } \\
\text { y Taylor, M. (2007) }\end{array}$ & $\begin{array}{l}\text { Academy of } \\
\text { Management } \\
\text { Review }\end{array}$ & $\begin{array}{l}\text { ¿En qué consiste la } \\
\text { creación de valor? }\end{array}$ & $\begin{array}{l}\text { Define la creación de valor en términos del valor de uso y de } \\
\text { intercambio. }\end{array}$ & 638 \\
\hline $\begin{array}{l}\text { Mahoney, J. y } \\
\text { Pandian, J. (1992) }\end{array}$ & $\begin{array}{l}\text { Strategic } \\
\text { Management } \\
\text { Journal }\end{array}$ & $\begin{array}{l}\text { ¿Cómo se relaciona el } \\
\text { enfoque de recursos } \\
\text { y capacidades con } \\
\text { otras perspectivas de } \\
\text { administración estratégica? }\end{array}$ & $\begin{array}{l}\text { El artículo plantea y desarrolla relaciones teóricas entre el RBV y } \\
\text { los enfoques de economía organizacional y organización industrial. }\end{array}$ & 1532 \\
\hline March, J. (1991) & $\begin{array}{l}\text { Organization } \\
\text { Science }\end{array}$ & $\begin{array}{l}\text { ¿Qué relación existe } \\
\text { entre la exploración de } \\
\text { nuevas posibilidades y la } \\
\text { explotación de capacidades } \\
\text { existentes para el } \\
\text { aprendizaje organizacional? }\end{array}$ & $\begin{array}{l}\text { Propone un modelo de aprendizaje mutuo (organizaciones-individuos) } \\
\text { y sus características. }\end{array}$ & ND \\
\hline
\end{tabular}


Tabla A1. Cuadro resumen revisión de literatura

\begin{tabular}{|c|c|c|c|c|}
\hline Autor & Journal & Pregunta & Contribución & Citaciones (*) \\
\hline $\begin{array}{l}\text { March, J. y Sutton, } \\
\text { R. (1997) }\end{array}$ & $\begin{array}{l}\text { Organization } \\
\text { Science }\end{array}$ & $\begin{array}{l}\text { ¿Por qué hay diferencias } \\
\text { en el desempeño de las } \\
\text { empresas? }\end{array}$ & $\begin{array}{l}\text { Identifica la necesidad de mejorar la comprensión del desempeño } \\
\text { desde la revisión de los métodos de investigación. }\end{array}$ & 452 \\
\hline $\begin{array}{l}\text { Moliterno, T. y } \\
\text { Wiersema, M. (2007) }\end{array}$ & $\begin{array}{l}\text { Strategic } \\
\text { Management } \\
\text { Journal }\end{array}$ & $\begin{array}{l}\text { ¿Cómo operan las } \\
\text { capacidades dinámicas? }\end{array}$ & $\begin{array}{l}\text { Explora la capacidad de desinversión y transferencia de recursos } \\
\text { entre empresas de una industria; lo cual implica el análisis de } \\
\text { generación y apropiación de rentas. }\end{array}$ & 122 \\
\hline Newbert, S. (2008) & $\begin{array}{l}\text { Strategic } \\
\text { Management } \\
\text { Journal }\end{array}$ & $\begin{array}{l}\text { ¿Cuáles son las relaciones } \\
\text { entre valor, ventaja } \\
\text { competitiva y desempeño? }\end{array}$ & $\begin{array}{l}\text { Los resultados del estudio cuantitativo que presenta el artículo } \\
\text { sugieren que el valor y la singularidad están relacionados con la } \\
\text { ventaja competitiva, que la ventaja competitiva está relacionada con } \\
\text { el rendimiento, y que la ventaja competitiva media la relación entre } \\
\text { singularidad y desempeño. }\end{array}$ & 393 \\
\hline $\begin{array}{l}\text { Nonaka, I., Toyama, } \\
\text { R. y Konno, N. } \\
(2000)\end{array}$ & $\begin{array}{l}\text { Long Range } \\
\text { Planning Journal }\end{array}$ & $\begin{array}{l}\text { ¿Qué es conocimiento? } \\
\text { ¿Cómo se origina y } \\
\text { evoluciona? ¿Cuál es } \\
\text { el papel del líder? }\end{array}$ & $\begin{array}{l}\text { Revela un modelo conceptual que muestra la dinámica de creación y } \\
\text { evolución del conocimiento en las organizaciones. }\end{array}$ & 1976 \\
\hline $\begin{array}{l}\text { Norman, W. y } \\
\text { Macdonald, C. } \\
(2004)\end{array}$ & $\begin{array}{l}\text { Business Ethics } \\
\text { Quarterly }\end{array}$ & $\begin{array}{l}\text { “La intención de 3BL es } \\
\text { llevar los paradigmas de la } \\
\text { contabilidad a los dominios } \\
\text { sociales y ambientales? ¿Es } \\
\text { esto una posibilidad práctica? } \\
\text { ¿Conseguirá esto los } \\
\text { objetivos previstos por los } \\
\text { promotores de 3BL? ¿O la } \\
\text { idea de un "resultado final” } \\
\text { en estos otros dominios es } \\
\text { una mera metáfora? Y si } \\
\text { es una metáfora, ¿es útil? } \\
\text { ¿Es esta una forma de jerga } \\
\text { que deberíamos adoptar y } \\
\text { fomentar?” }\end{array}$ & $\begin{array}{l}\text { Síntesis de enfoques de medición del impacto de las organizaciones } \\
\text { más allá de la cuenta de resultados contable. Revela interés por } \\
\text { evidenciar las externalidades que las empresas generan en el medio } \\
\text { ambiente y la sociedad, y la forma de evidenciarlo y reportarlo como } \\
\text { resultado formal de las empresas. }\end{array}$ & 428 \\
\hline $\begin{array}{l}\text { Peteraf, M. y Barney, } \\
\text { J. (2003) }\end{array}$ & $\begin{array}{l}\text { Managerial } \\
\text { and Decision } \\
\text { Economics }\end{array}$ & $\begin{array}{l}\text { ¿Cuáles son los dominios } \\
\text { de la perspectiva de } \\
\text { recursos y capacidades? }\end{array}$ & $\begin{array}{l}\text { Proporciona una definición de ventaja competitiva, alineada con la } \\
\text { noción de generación de valor, la cual reconoce el papel que cumplen } \\
\text { los recursos VRIN en la generación de las diferentes rentas. }\end{array}$ & 658 \\
\hline Porter, M. (1991) & $\begin{array}{l}\text { Strategic } \\
\text { Management } \\
\text { Journal }\end{array}$ & $\begin{array}{l}\text { ¿Cuáles son las causas de el } \\
\text { desempeño superior en un } \\
\text { periodo de tiempo? ¿Cuál } \\
\text { es el proceso dinámico } \\
\text { en el cual las posiciones } \\
\text { competitivas son creadas? }\end{array}$ & $\begin{array}{l}\text { Identifica las fuentes de ventaja en un ambiente competitivo y las } \\
\text { condiciones que influencian las decisiones gerenciales en una } \\
\text { industria determinada. }\end{array}$ & 1691 \\
\hline Porter, M. (1981) & $\begin{array}{l}\text { Academy of } \\
\text { Management } \\
\text { Review }\end{array}$ & $\begin{array}{l}\text { ¿Cuáles son las } \\
\text { contribuciones de la } \\
\text { perspectiva de organización } \\
\text { industrial a la administración } \\
\text { estratégica? }\end{array}$ & $\begin{array}{l}\text { Revela una síntesis de autores clásicos que fundamentan el estudio en } \\
\text { el campo de la estrategia. }\end{array}$ & ND \\
\hline $\begin{array}{l}\text { Porter, M. y Kramer, } \\
\text { M. (2011) }\end{array}$ & $\begin{array}{l}\text { Harvard } \\
\text { Business Review }\end{array}$ & $\begin{array}{l}\text { ¿Cómo reinventar el } \\
\text { capitalismo y desatar } \\
\text { una ola de innovación y } \\
\text { crecimiento? }\end{array}$ & $\begin{array}{l}\text { El artículo introduce el concepto de valor compartido como un } \\
\text { conjunto de políticas y prácticas operacionales que incrementa la } \\
\text { competitiividad de la empresa al tiempo que considera la relación } \\
\text { con actores sociales y comunidades en las que se opera. }\end{array}$ & 2647 \\
\hline Priem, R. (2007) & $\begin{array}{l}\text { Academy of } \\
\text { Management } \\
\text { Review }\end{array}$ & $\begin{array}{l}\text { ¿Cómo las estrategias de las } \\
\text { empresas que se centran en } \\
\text { la mejora de los beneficios } \\
\text { para el consumidor pueden } \\
\text { crear valor aumentando la } \\
\text { retribución a todo el sistema } \\
\text { de valor? }\end{array}$ & $\begin{array}{l}\text { Mediante el enfoque Consumer Benefit Experienced el autor establece } \\
\text { diferencias entre el concepto valor creado y valor capturado. }\end{array}$ & 382 \\
\hline
\end{tabular}

Richard, P.,

Devinney, T., Yip, G.

y Johnson, G. (2009)
Journal of Management
¿Cómo medir

el desempeño organizacional?
Establece las métricas más utilizadadas en la investigación sobre desempeño en administración estratégica y precisa la heterogeneidad de productos en diferentes condiciones de mercado, grupos de interés y comportamiento diferenciado en el tiempo como dimensiones predominantes.
(Continúa)

106

Multidiscip. Bus. Rev. | Vol. 14, $N^{\circ} 1$, 2021, pp. 95-107, ISSN 0718-400X

DOI: https://doi.org/10.35692/07183992.14.1.9 
Tabla A1. Cuadro resumen revisión de literatura

\begin{tabular}{|c|c|c|c|c|}
\hline Autor & Journal & Pregunta & Contribución & Citaciones (*) \\
\hline $\begin{array}{l}\text { Rumelt, R., } \\
\text { Schendel, D. y Teece, } \\
\text { D. (1991) }\end{array}$ & $\begin{array}{l}\text { Strategic } \\
\text { Management } \\
\text { Journal }\end{array}$ & $\begin{array}{l}\text { ¿Por qué hablar } \\
\text { de economía en } \\
\text { administración estratégica? }\end{array}$ & $\begin{array}{l}\text { Identifica cinco eventos sobre los cuales gira la relación entre las dos } \\
\text { disciplinas y traza líneas de investigación futura. }\end{array}$ & 441 \\
\hline $\begin{array}{l}\text { Smith, J. y Colgate, } \\
\text { M. (2007) }\end{array}$ & $\begin{array}{l}\text { Journal of } \\
\text { Marketing } \\
\text { Theory and } \\
\text { Practice }\end{array}$ & $\begin{array}{l}\text { ¿Qué es la creación de } \\
\text { valor para el cliente? }\end{array}$ & $\begin{array}{l}\text { Propone un marco conceptual para la diferenciación del valor que } \\
\text { percibe el cliente en el mercado. }\end{array}$ & 378 \\
\hline $\begin{array}{l}\text { Smith, M. y Bititci, } \\
\text { U. (2017) }\end{array}$ & $\begin{array}{l}\text { International } \\
\text { Journal of } \\
\text { Operations and } \\
\text { Production } \\
\text { Management }\end{array}$ & $\begin{array}{l}\text { ¿Cuál es la relación entre } \\
\text { desempeño, medición } \\
\text { del desempeño, sistema } \\
\text { de administración del } \\
\text { desempeño y compromiso } \\
\text { del personal? }\end{array}$ & $\begin{array}{l}\text { Muestra que una intervención en los controles sociales da lugar a } \\
\text { cambios en el sistema de medición y gestión del desempeño, lo que } \\
\text { genera una mejora significativa en el compromiso y desempeño de } \\
\text { los colaboradores en la organización. }\end{array}$ & 35 \\
\hline $\begin{array}{l}\text { Teece, D., Pisano, G. } \\
\text { y Shuen, A. (1997) }\end{array}$ & $\begin{array}{l}\text { Strategic } \\
\text { Management } \\
\text { Journal }\end{array}$ & $\begin{array}{l}\text { ¿Cómo las empresas } \\
\text { logran y mantienen una } \\
\text { ventaja competitiva? }\end{array}$ & $\begin{array}{l}\text { El marco sugiere que la creación de riqueza privada en regímenes } \\
\text { de cambio tecnológico rápido depende en gran medida del } \\
\text { perfeccionamiento de los procesos tecnológicos, organizativos y de } \\
\text { gestión internos de la empresa. }\end{array}$ & 13769 \\
\hline $\begin{array}{l}\text { Venkatraman, N. } \\
\text { y Ramanujam, V. } \\
\text { (1986) }\end{array}$ & $\begin{array}{l}\text { Academy of } \\
\text { Management } \\
\text { Review }\end{array}$ & $\begin{array}{l}\text { ¿Cuáles son los enfoques de } \\
\text { medición del desempeño en } \\
\text { estrategia? }\end{array}$ & $\begin{array}{l}\text { Identifica tres dominios del desempeño (finanzas, operaciones } \\
\text { y organización) y diez diferentes enfoques de la medición de } \\
\text { desempeño contrastando las dimensiones de conceptualización y } \\
\text { fuentes de información. }\end{array}$ & 570 \\
\hline Wernerfelt, B. (1984) & $\begin{array}{l}\text { Strategic } \\
\text { Management } \\
\text { Journal }\end{array}$ & $\begin{array}{l}\text { ¿Son los recursos y su } \\
\text { asignación fuente de ventaja } \\
\text { competitiva? }\end{array}$ & $\begin{array}{l}\text { Propone el análisis desde la perspectiva de los recursos y para ello } \\
\text { introduce el concepto de resource-position barrier y resource- } \\
\text { product matrix. }\end{array}$ & 10976 \\
\hline Zott, C. (2003) & $\begin{array}{l}\text { Strategic } \\
\text { Management } \\
\text { Journal }\end{array}$ & $\begin{array}{l}\text { ¿Cómo las capacidades } \\
\text { dinámicas de una firma } \\
\text { están relacionadas con el } \\
\text { diferencial de desempeño } \\
\text { industrial? }\end{array}$ & $\begin{array}{l}\text { Se propone un modelo formal en el que las capacidades dinámicas } \\
\text { se tratan como un conjunto de rutinas que guían la evolución de la } \\
\text { configuración de los recursos de una empresa. }\end{array}$ & 609 \\
\hline
\end{tabular}

(*) Fuente: elaboración propia con base en revisión de literatura. Base de datos Scopus para citación. 